\title{
The Value of Component Commonality in a Dynamic Inventory System with Lead Times
}

\author{
Jing-Sheng Song • Yao Zhao \\ The Fuqua School of Business, Duke University, Durham, NC 27708 \\ Dept of Supply Chain Management \& Marketing Sciences, Rutgers University, Newark, NJ 07102 \\ jssong@duke.edu•yaozhao@andromeda.rutgers.edu
}

June 23, 2008

\begin{abstract}
Component commonality has been widely recognized as a key factor in achieving product variety at low cost. Yet, the theory on the value of component commonality is rather limited in the inventory literature. The existing results were built primarily on single-period models or periodic-review models with zero lead times. In this paper, we consider a continuous-review system with positive lead times. We find that while component commonality is in general beneficial, its value depends strongly on component costs, lead times and dynamic allocation rules. Under certain conditions, several previous findings based on static models do not hold here. In particular, component commonality does not always generate inventory benefits under certain commonly used allocation rules. We provide insight on when component commonality generates inventory benefits and when it may not. We further establish some asymptotic properties that connect component lead times and costs to the impact of component commonality. Through numerical studies, we demonstrate the value of commonality and its sensitivity to various system parameters in between the asymptotic limits. In addition, we show how to evaluate the system under a new allocation rule, a modified version of the standard FIFO rule.
\end{abstract}

Key words: Component commonality, dynamic model, lead times, allocation rules, assemble-toorder systems 


\section{Introduction}

Component commonality has been widely recognized as an important way to achieve product variety at low cost. It is one of the key preconditions for mass customization, such as Assemble-toOrder systems. Examples can be found in many industries, including computers, electronics and automobiles; see Ulrich (1995), Whyte (2003) and Fisher, et al. (1999). To implement component commonality, companies must decide to what extent they should standardize components and modularize subassemblies so that they can be shared among different products. It is not surprising, then, that the topic of component commonality has drawn lots of attention from researchers. For recent literature reviews, see, Krishnan and Ulrich (2001), Ramdas (2003) and Song and Zipkin (2003). While component commonality has substantial impact on issues such as product development, supply chain complexity and production costs, see e.g., Desai et al. (2001), Fisher et al. (1999), Gupta and Krishnan (1999) and Thonemann and Brandeau (2000), our focus here is its impact on inventory-service tradeoffs.

In particular, we compare optimal inventory investments before and after using common components while keeping the level of product availability (fill rate) constant in both systems. We identify conditions under which component sharing leads to greater inventory savings. This kind of analysis can help managers evaluate the tradeoffs of component costs before and after standardization. Our work follows those pioneered by Collier (1981, 1982), Baker (1985), Baker, et al. (1986) and extended by many others, such as Gerchak, et al. (1988), Gerchak and Henig (1989), Bagchi and Gutierrez (1992), Eynan (1996), Eynan and Rosenblatt (1996), Hillier (1999), Swaminathan and Tayur (1998) and Van Mieghem (2004). These studies typically show that component commonality is always beneficial and can be significant when the number of products increases, demand correlation decreases and the degree of commonality increases.

So far, the existing results in this line of research, including the papers mentioned above, have been built primarily on static models (either single-period models or multi-period, periodic-review models with zero lead times); see Song and Zipkin (2003) and Van Mieghem (2004) for recent reviews. Our study makes an effort to push this research forward to consider dynamic models with positive lead times.

The issue (value of component commonality) turns out to be surprisingly delicate. Intuitively, as something shared by many products, a common component allows us to explore the effect of risk pooling - a property well known for the role of a central warehouse in the one-warehouse, multiple- 


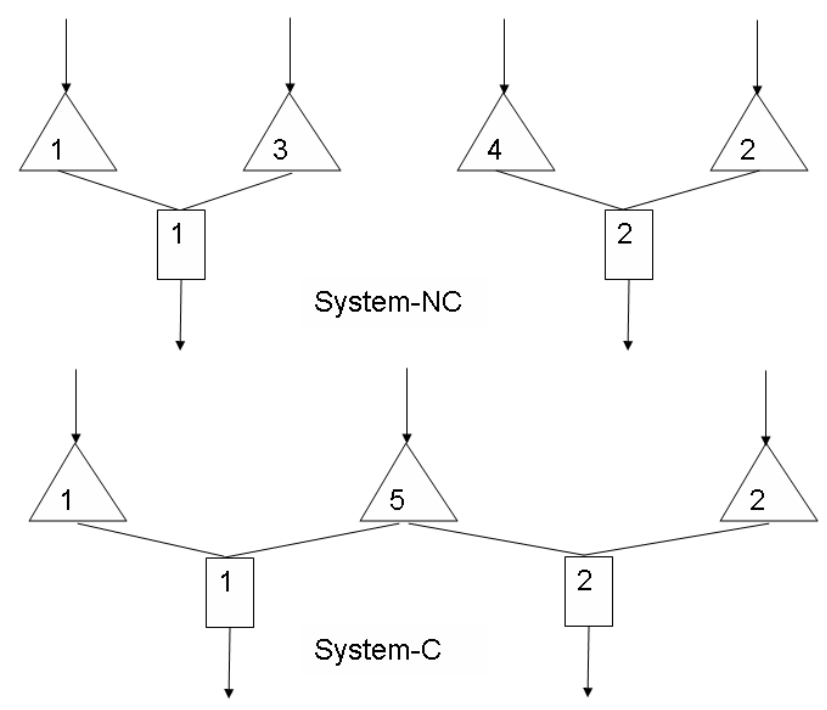

Figure 1: The two-product ATO systems.

retailer distribution system design context. That is, by aggregating individual retailer demand, we can reduce overall demand uncertainty (at the central warehouse) and in doing so, reduce safety stock requirements for the entire system. However, unlike in the distribution network setting, the topology of product structures with component commonality also contains the assembly feature the availability of both common and product-specific components affects demand fulfillment. Thus, the value of component commonality is more intricate than the risk pooling effect associated with a central warehouse in a distribution network.

To gain insight into this complex scenario, researchers have employed a stylized two-product ATO system in which each product is assembled from two components, as illustrated in Figure 1. In the first setting, products are assembled from unique components and there is no common component, so it is called "System-NC." In the second setting, components 1 and 2 are still product specific for products 1 and 2, respectively, but components 3 and 4 are replaced by a common component 5. We call this setting "System-C."

Let $c_{i}$ be unit cost and $L_{i}$ be the lead time for component $i, i=1,2, \ldots, 5$. In System-NC, let $s_{i}$ be the base-stock level of component $i, i=1,2,3,4$, and use $\underline{s}$ to denote $\left\{s_{1}, s_{2}, s_{3}, s_{4}\right\}$. In System-C, let $\tilde{s}_{i}, i=1,2,5$ be the base-stock level of component $i$, and use $\underline{\tilde{s}}$ to denote $\left\{\tilde{s}_{1}, \tilde{s}_{2}, \tilde{s}_{5}\right\}$. Let $\mathcal{Z}_{+}^{n}$ denote $n$-dimensional space of nonnegative integers. Then $\underline{s} \in \mathcal{Z}_{+}^{4}$ and $\underline{\tilde{s}} \in \mathcal{Z}_{+}^{3}$.

The objective is to minimize total inventory investment while satisfying the desired fill rate for each product. Let $\alpha^{j}$ be the target fill rate for product $j$. Denote by $f^{j}$ the fill rate of product $j$ in System-NC, and let $\tilde{f}^{j}$ be its counterpart in System-C. Assuming that the system takes ownership 
of goods at the time that the order is placed, we have the following mathematical programs for the two systems:

$$
\begin{aligned}
& \text { Problem-NC } \quad C^{*}= \min \quad \sum_{i=1}^{4} c_{i} s_{i} \\
& \text { s.t. } \quad f^{j}(\underline{s}) \geq \alpha^{j}, j=1,2, \quad \underline{s} \in \mathcal{Z}_{+}^{4}, \\
& \text { Problem-C } \quad \tilde{C}^{*}=\min \quad \sum_{i=1,2} c_{i} \tilde{s}_{i}+c_{5} \tilde{s}_{5} \\
& \\
& \text { s.t. } \quad \tilde{f}^{j}(\underline{\tilde{s}}) \geq \alpha^{j}, j=1,2, \quad \underline{\tilde{s}} \in \mathcal{Z}_{+}^{3} .
\end{aligned}
$$

Baker et al. (1986) studied this problem using a single-period model with uniformly distributed product demands. Their results are later extended by Gerchak et al. (1988) and Gerchak and Henig (1989) to multi-product, multi-period systems with general demand. Differently from ours, these works study periodic-review systems with zero lead times $\left(L_{i}=0\right.$ for all $\left.i\right)$. Demand in each period is batched and fulfilled all at once at the end of the period. Unfulfilled demand is either lost or backlogged but can be cleared at the beginning of the next period because the lead times are zero. Let $s_{i}^{*}, i=1,2,3,4\left(\tilde{s}_{i}^{*}, i=1,2,5\right)$ be the optimal solution to Problem-NC (Problem-C, respectively). Under the assumption of $c_{3}=c_{4}=c_{5}$, their main results are:

P1 $\tilde{C}^{*} \leq C^{*}$. That is, the introduction of commonality reduces the total inventory investment required to maintain a specific service level.

P2 If $c_{1}=c_{2}$, then $\tilde{s}_{5}^{*} \leq s_{3}^{*}+s_{4}^{*}$. That is, when the product-specific components have identical costs, the optimal stock level of the common component is lower than the combined optimal stock levels it replaces.

P3 If $c_{1}=c_{2}$, then $\tilde{s}_{i}^{*} \geq s_{i}^{*}, i=1,2$. That is, when the product-specific components have identical costs, the combined optimal stock levels of product-specific components are higher with commonality than without it.

A natural question arises at this point: will these results still hold in dynamic models with lead times? If component costs and lead times are the same before and after component sharing, then it can be shown that the optimal policy of System-NC is always feasible for System-C (i.e., satisfies the fill rate constraints). This is true because such a policy is a special case of those of System-C which partition the inventory of a common component into separate bins, each committed to a product. Therefore, the optimal inventory investment of System-C should be no greater than that 
of System-NC. The problem is, the optimal policy of System-C is not known and therefore we do not know how to achieve its optimal cost.

The control of an ATO system consists of two decisions: one is inventory replenishment and the other is inventory allocation. If the objective is to minimize long-run inventory holding and backorder penalty costs, then the optimal decisions are generally state-dependent; see Song and Zipkin (2003). Recently, Benjaafar and ElHafsi (2006) consider a special case: an assembly system with a single end-product and multiple demand classes. Each component supplier has a finite production capacity. They show that under Markovian assumptions, the optimal replenishment policy is a state-dependent base-stock policy, and the optimal allocation rule is a multi-level rationing policy that depends on the inventory levels of all other components. Obviously, the optimal policy for a general multi-product ATO system is even more complex.

For these reasons, only simple but suboptimal replenishment and allocation policies are implemented in practice. When a suboptimal policy is used, it is not obvious whether component commonality can always yield inventory savings. Indeed, Song (2002) examines a model with constant lead times following an independent base-stock policy and the first-in-first-out (FIFO) stock allocation rule. In a numerical study, she shows that with the same level of inventory investment, component sharing can result in more product backorders. This observation motivated us to analyze this issue more thoroughly.

Consistent with practice, we focus on some simple but commonly used suboptimal policies. First, we assume that state-independent base-stock policies are employed for inventory replenishment. In the continuous-review system we consider, this policy implies that a replenishment order is placed for each required component at each demand arrival - the so-called one-for-one replenishment policy. Second, we consider two component allocation rules - the first-in-first-out (FIFO) rule and a modified first-in-first-out (MFIFO) rule. We describe them in detail below.

Under FIFO, demand for each component is fulfilled in exactly the same sequence as it occurs. This is a component-based allocation rule, because the allocation of one component's inventory does not depend on the inventory status of other components. When a product demand arrives, if some of its components are available while others are not, the available components are put aside as committed stock. When a component is replenished, we commit it to the oldest backordered product that requires it. This rule is suboptimal because it commits available components to backlogged demand, creating extra waiting time for both the available components and for other demand. The virtue of this rule is simplicity of its information requirements and implementation. 
Moreover, it is perceived to be fair to the customers.

Under MFIFO, product demand is filled following the FIFO rule as long as all components demanded are available. When a product demand arrives and some of its components are not available, however, we assign the demand to the last position in the backorder list and do not allocate or commit those available components to the order. That is, a unit of component inventory will not be allocated to an incoming order, unless such an allocation results in the completion of the order fulfillment. When a replenishment shipment arrives, we review the backorder list and satisfy the oldest backorder for which all required components are available. This is a product-based allocation rule; the allocation of one component's inventory to a demand depends on the inventory status of other components. Consequently, it requires more information and more coordination than FIFO. It also may make some orders wait longer than under FIFO.

Note that we consider the total inventory investment (in the base-stock levels) as the objective function in this paper. Although it is a common choice in the inventory literature, the objective function does not account for the extra holding costs charged in the FIFO system for the committed components. Including these costs would affects the economics of the FIFO allocation rule adversely.

Both FIFO and MFIFO are commonly seen in practice. Kapuscinski, et al. (2004) describe an example of MFIFO at Dell, and Cheng et al. (2002) describe an example of FIFO at IBM.

$\S 3$ focuses on the FIFO system while $\S 4$ analyzes the system under MFIFO. To the best of our knowledge, our analysis provides the first closed-form evaluation of an allocation rule more complex than FIFO. We also show that the closed-form evaluation works for a class of no-holdback allocation rules that do not holdback on-hand inventory when backorders exist, which includes MFIFO as a special case. In addition, we show that, for any given base-stock policy, MFIFO achieves the same product fill rates as any other no-holdback rule. So the results on MFIFO apply to all no-holdback rules.

Equipped with the analytical tools, we show that, in the case of equal lead times across components, properties P1-P3 hold under MFIFO, but properties P1-P2 do not hold under FIFO. In the case of unequal lead times, properties P1-P3 fail to hold under both FIFO and MFIFO. Thus, under these commonly used allocation rules, component commonality does not always provide inventory benefits. Our analysis further sheds light on why this could happen. One important implication of these observations is that product structure (i.e., making several products share some common components) alone may not necessarily lead to inventory savings. We also need an effective operational policy specially tailored to the new product structure. 
To identify when component commonality is more likely to yield inventory benefit under the simple control policies considered here, and to quantify such benefit, in $\S 5$ we perform an asymptotic analysis on the system parameters. The analysis applies to both FIFO and MFIFO. We also conduct an extensive numerical study in $\S 6$ to examine the value of commonality when the parameters are in between the asymptotic limits. In general, we find that component sharing is beneficial under these simple policies. The resulting inventory savings is more significant under MFIFO or when the common component is more expensive or has a longer lead time than the product-specific components. At an extreme, when the costs of the product-specific components are negligible relative to that of the common component, the value of the component commonality converges to the risk pooling effect associated with a central warehouse in the distribution network setting. This is the maximum inventory benefit component commonality can achieve. We also find that the performance gap between MFIFO and FIFO tends to be higher as the target fill rate decreases or when the common component has a shorter lead time than the product-specific components. Finally, in $\S 7$, we summarize the insights and conclude the paper.

\section{Other Related Literature}

In the literature of dynamic ATO systems with positive lead times, most papers assume independent base-stock replenishment policies; see Song and Zipkin (2003) for a survey. For continuous-review models (which is the setting in this paper), to our knowledge, all papers consider the FIFO allocation rule; see, for example, Song (1998, 2002), Song, et al. (1999), Song and Yao (2002), and Lu, et al. (2003, 2005), and Zhao and Simchi-Levi (2006). For periodic-review models, because the demands during each period are batched and filled at the end of the period, different allocation rules have been considered. These include a fixed priority rule by Zhang (1997), the FIFO rule by Hausman, et al. (1998), a fair-share rule by Agrawal and Cohen (2001), and a product-based allocation rule by Akcay and Xu (2004). However, all these studies apply FIFO for demands between periods. In other words, if implemented in a continuous-review environment, these alternative allocation rules reduce to FIFO. The policy choices in the asymptotic studies by Plambeck and Ward (2003, 2005) on high-volume ATO systems are exceptions, but their setting is quite different from ours. There, the decision variables include the component production capacities, product production sequence, and product prices. Once a component's production capacity is chosen, the production facility produces the component at full capacity, so there is no inventory decision.

In addition to Song (2002), several other authors conduct numerical experiments to study the 
value of component commonality in systems with lead times, using the analytical tools developed in their papers. For example, Agrawal and Cohen (2001) consider periodic-review assembly systems with constant lead times. Component inventories are managed by base-stock policies and common components are allocated according to the fair-share rule. Assuming that the demands for components follow a multi-variate normal distribution, the authors study a four-product, two-component example numerically and show that a higher degree of commonality leads to lower costs. Cheng, et al. (2002) study configure-to-order (CTO) systems based on real world applications from IBM. Common components are allocated according to the FIFO rule, and component replenishment lead times are stochastic. The authors develop an approximation for order-based service levels. Using numerical studies, they show that for given service levels, up-to $25.8 \%$ reduction of component inventory investments can be achieved by sharing components, and the savings increase as the number of associated products increases. Our analytical results are consistent with the observations of these studies.

It is worth mentioning that Alfaro and Corbett (2003) also point out that the effect of pooling may be negative when the inventory policy in use is suboptimal. However, their study focuses on a single component only, analogous to the warehouse setting. Thus, they do not consider the assembly operation. Moreover, they use a single-period model, so component lead time is not an issue. Finally, in their setting, the form of the optimal policy is known, and they use suboptimal policy parameters to conduct their study. In our setting, the form of the optimal policy is unknown, so we focus on a class of suboptimal policies that is widely used in practice. But, within this class of policies, our study assumes that the policy parameters are optimized.

Rudi (2000) studies the impact of component cost on the value of commonality in a single-period model. He shows qualitatively that a more expensive common component leads to higher savings from commonality. Our work complements his study by examining a dynamic system with positive lead times and quantifying the component-cost effect through asymptotic results and numerical examples.

It is common in the literature to approximate the value of component commonality by the risk-pooling effect associated with a central warehouse (Eppen 1979). See, for example, Chopra and Meindl (2001) pg. 204-205, Simchi-Levi, et al. (2003) pg. 223-224, and Thonemann and Brandeau (2000). This approximation, however, ignores the impact of the product-specific components and therefore may overestimate the benefits. In this paper, we develop insights on when this approximation is reasonably accurate, and when it is not. 
Finally, we note that Thonemann and Bradley (2002) conduct asymptotic analysis on the affect of setup times on the cost of product variety by letting the number of products approach infinity. Their focus, model setting, as well as the type of limit, are all different from ours.

\section{Analysis under FIFO}

This section focuses on the two-product ATO system described in $§ 1$ (see Figure 1) under the FIFO rule and discusses the validity of properties P1 - P3. This simple system connects well with the existing literature on static models without lead times. It also lays the foundation for the more general systems considered in $\S 5$.

We assume that demand for product $j$ forms a Poisson process (unit demand) with rate $\lambda^{j}$, $j=1,2$, and one unit of each component is needed to assemble one unit of final product. The inventory of each component is controlled by an independent continuous-time base-stock policy with an integer base-stock level. Replenishment lead times of components are constant but not necessarily identical.

\subsection{Fill Rate Comparison}

To compare the solutions of Problem-NC with Problem-C under the FIFO rule, it is important to understand the relationship between fill rates $f^{j}$ and $\tilde{f}^{j}$. By Song (1998), for $j=1,2$,

$$
\begin{aligned}
& f^{j}=\operatorname{Pr}\left\{D_{j}\left(L_{j}\right)<s_{j}, D_{2+j}\left(L_{2+j}\right)<s_{2+j}\right\} \\
& \tilde{f}^{j}=\operatorname{Pr}\left\{D_{j}\left(L_{j}\right)<\tilde{s}_{j}, D_{5}\left(L_{5}\right)<\tilde{s}_{5}\right\}
\end{aligned}
$$

where $D_{i}\left(L_{i}\right), i=1,2, \ldots, 5$, are the lead time demand for components. Let $D^{j}(L)$ be the demand for product $j$ during time $L$. Then,

$$
\begin{aligned}
& D_{i}(L)=D_{2+i}(L)=D^{i}(L), \quad i=1,2, \\
& D_{5}(L)=D^{1}(L)+D^{2}(L) .
\end{aligned}
$$

To examine properties P1-P3, we assume that both the common component 5 and the components that it replaces have the same cost and lead time, i.e., $c_{i}=c^{\prime}$ and $L_{i}=L^{\prime}, i=3,4,5$. The following proposition shows that the optimal solution in System-NC may not be feasible in System-C under the FIFO rule. 
Proposition 1 Assume the same lead time across all components, i.e., $L_{1}=L_{2}=L$ and $L^{\prime}=L$. If the base-stock levels of the product-specific components are the same in System-NC and System-C, i.e., $\tilde{s}_{i}=s_{i}^{*}, i=1,2$, then,

(a) the order-based fill rates in System-NC are greater than their counterparts in System-C for any finite $\tilde{s}_{5}$. That is,

$$
f^{j}>\tilde{f}^{j}, \quad j=1,2 .
$$

(b) The solution $\underline{\tilde{s}}=\left\{s_{1}^{*}, s_{2}^{*}, s_{3}^{*}+s_{4}^{*}\right\}$ may not be feasible for System-C.

Proof. By $L^{\prime}=L$ and Eq. (5), the optimal base-stock levels in System-NC must satisfy

$$
s_{j}^{*}=s_{2+j}^{*}, j=1,2 .
$$

For any finite integer $\tilde{s}_{5}$ and $j=1,2$, by Eqs. (3)-(6) and (8),

$$
\begin{aligned}
f^{j} & =\operatorname{Pr}\left\{D^{j}(L)<s_{j}^{*}, D^{j}(L)<s_{2+j}^{*}\right\} \\
& =\operatorname{Pr}\left\{D^{j}(L)<s_{j}^{*}\right\} \\
& =\operatorname{Pr}\left\{D^{j}(L)<\tilde{s}_{j}\right\} \\
& >\operatorname{Pr}\left\{D^{j}(L)<\tilde{s}_{j}, D^{1}(L)+D^{2}(L)<\tilde{s}_{5}\right\} \\
& =\tilde{f}^{j}
\end{aligned}
$$

proving (a). Note $\alpha^{j}$ can choose any real number in $(0,1)$, so Part (b) is a direct consequence of Part (a).

Proposition 1 (a) offers some interesting insights. Note that the first equality in (9) holds because $D_{j}(L)=D_{2+j}(L)=D^{j}(L)$ in any event (Eq. 5). So, with equal component lead times, the supplies of the two components for product $j$ in System-NC are completely synchronized. Replacing components 3,4 by a common component 5 disrupts this synchronization: because $D_{5}(L)$ includes the demand for all products, it is only partially correlated with $D_{j}(L), j=1,2$. In other words, the supply process of the common component is only partially correlated with the supply process of the product-specific component. Because of the assembly feature, it is then intuitive that the less synchronized component supply processes in System-C result in smaller order-based fill rates. (Indeed, inequality (9) provides an analytical explanation for the numerical observation of Song (2002) mentioned in $\S 1)$.

In a single-period model or periodic review systems with zero lead times, if one keeps the stock levels of the product-specific components unchanged and lets the stock level of the common component be the sum of stock levels of those it replaces, the optimal solution without component 
sharing is always feasible for the corresponding component sharing system (see, e.g., Baker et al. 1986). Proposition 1 (b) indicates that this statement does not hold in dynamic systems with lead times under the FIFO rule.

\subsection{Counterexamples to P1-P3}

In this section, we show that while P3 holds for the equal lead time case, it fails to hold for the unequal lead time case. We also show that P1 and P2 do not hold even for the equal lead time case.

Observation 2 Assume the same lead time across all components. Then $\mathbf{P 3}$ holds under FIFO. That is, the optimal product-specific base-stock levels in System-C are no less than those in System$N C$.

Proof. See appendix for details.

Observation 3 Assume the same lead time across all components. Then $\mathbf{P} 1$ and $\mathbf{P} 2$ do not hold. That is, for the same target service levels, System-C may require higher optimal inventory investment than System-NC, so component commonality does not always lead to inventory benefit. Also, the optimal stock level of the common component may exceed the combined stock levels it replaces.

Proof. It suffices to construct numerical counterexamples to $\mathbf{P 1}$ and $\mathbf{P 2}$. Considering a symmetric two-product system with $L=L^{\prime}, c_{1}=c_{2}=c, c^{\prime}=1, \alpha^{j}=\alpha, \lambda^{j}=1, j=1,2$. Due to symmetry, we only need to consider product 1 and its associated components, 1, 3 and 5 . Numerical results are summarized in Table 1, where the percentage savings refers to the relative reduction of the total inventory investment from Problem-NC to Problem-C, i.e., $100 *\left(C^{*}-\tilde{C}^{*}\right) / C^{*}$. We use enumeration based on Eqs. (3)-(4) to obtain the optimal solutions for Problem-NC and Problem-C.

Table 1: Counterexamples to $\mathbf{P 1}$ and $\mathbf{P 2}$ under FIFO

\begin{tabular}{|c|c|c|c|c|c|c|c|c|}
\hline$\alpha$ & $L$ & $c / c^{\prime}$ & $\%$ Savings & $\begin{array}{c}\text { soln. (NC) } \\
\left(s_{1}^{*}, s_{3}^{*}\right)\end{array}$ & fill rate (NC) & $\begin{array}{c}\text { soln. (C) } \\
\left(\tilde{s}_{1}^{*}, \tilde{s}_{5}^{*}\right)\end{array}$ & fill rate (C) & $\begin{array}{c}\text { fill rate (C) } \\
\tilde{s}_{1}=s_{1}^{*}, \tilde{s}_{5}=s_{3}^{*}+s_{4}^{*}\end{array}$ \\
\hline $95 \%$ & 10 & 5 & $-1.04 \%$ & $(16,16)$ & $95.13 \%$ & $(16,34)$ & $95.06 \%$ & $94.84 \%$ \\
\hline $95 \%$ & 10 & 10 & $-0.57 \%$ & $(16,16)$ & $95.13 \%$ & $(16,34)$ & $95.06 \%$ & $94.84 \%$ \\
\hline $95 \%$ & 10 & 50 & $-0.12 \%$ & $(16,16)$ & $95.13 \%$ & $(16,34)$ & $95.06 \%$ & $94.84 \%$ \\
\hline $70 \%$ & 1 & 1 & $-12.5 \%$ & $(2,2)$ & $73.5 \%$ & $(2,5)$ & $72.7 \%$ & $69.92 \%$ \\
\hline
\end{tabular}

In these numerical examples, $C^{*}<\tilde{C}^{*}$ and $\tilde{s}_{5}^{*}>2 s_{3}^{*}$. The first fact implies that $\mathbf{P} \mathbf{1}$ may not hold; the second fact indicates that $\mathbf{P 2}$ may not hold because $s_{3}^{*}+s_{4}^{*}=2 s_{3}^{*}$ due to symmetry. 
We now provide insight into the counterexamples. In the first three cases of Table $1, c / c^{\prime}$ is high $\left(c / c^{\prime}=5,10,50\right)$, i.e., the common component is inexpensive relative to product-specific components. If we set $\left(\tilde{s}_{1}, \tilde{s}_{5}\right)=(16,32)$, the fill-rate of System-C is $94.8 \%(<95 \%)$ (which is possible by Proposition 1). To achieve the target fill-rate $95 \%$ in System-C, we only have two options: (i) increasing $\tilde{s}_{i}$ from 16 for at least one product-specific component $i=1$, 2 , or (ii) keeping $\tilde{s}_{i}=16, i=1,2$ but increasing $\tilde{s}_{5}$ from 32 until the fill rate constraint(s) are satisfied. For convenience, we call option (i) the specific option and option (ii) the common option.

Clearly, the specific option increases the inventory investment of the product-specific component(s), while the common option only increases the inventory investment of the common component. Because $c^{\prime} \ll c$ in these cases, it turns out that the optimal solution here is to increase the base-stock level of the common component to 34 while keeping the base-stock levels of productspecific components unchanged. It is interesting to note that in these cases, the optimal solutions in Problem-NC not only have lower total inventory investments but also have higher fill-rates (95.13\%), than their counterparts in Problem-C (95.06\%).

The last case of Table 1 shows that counterexamples can occur even when the common component is not so inexpensive relative to the product-specific components.

Observation 4 Property $\mathbf{P 3}$ may not hold if $L_{1}=L_{2}=L$ but $L \neq L^{\prime}$. That is, when the common component has a different lead time from those of the product-specific components, the combined optimal stock levels of product-specific components may be lower in System-C than in System-NC, i.e., $\tilde{s}_{1}^{*}$ can be smaller than $s_{1}^{*}$. See Table 2 for an example, where $L_{1}=L_{2}=L=2, L^{\prime}=10$, $c_{1}=c_{2}=c=0.1, c^{\prime}=1, \alpha^{j}=95 \%, \lambda^{j}=1, j=1,2$.

Table 2: A counterexample to $\mathbf{P 3}$ under FIFO

\begin{tabular}{|c|c|c|c|c|}
\hline$c / c^{\prime}$ & $\begin{array}{c}\text { soln. (NC) } \\
\left(s_{1}^{*}, s_{3}^{*}\right)\end{array}$ & fill rate (NC) & $\begin{array}{c}\text { soln. (C) } \\
\left(\tilde{s}_{1}^{*}, \tilde{s}_{5}^{*}\right)\end{array}$ & fill rate (C) \\
\hline 0.1 & $(8,16)$ & $95.08 \%$ & $(6,29)$ & $95.19 \%$ \\
\hline
\end{tabular}

Intuitively, when $L \neq L^{\prime}, s_{1}^{*}$ may not be the smallest integer such that $\operatorname{Pr}\left\{D^{1}(L)<s_{1}^{*}\right\} \geq 0.95$. This is especially true when $c \ll c^{\prime}$, because in this case, the optimal solution in Problem-NC is to keep $s_{3}^{*}$ at the minimum while increasing $s_{1}$ until the solution is feasible. While in Problem-NC, the cost of increasing $s_{1}$ (or $\left.s_{3}\right)$ by one unit is $2 * c\left(2 * c^{\prime}\right.$ respectively); in Problem-C, the cost of increasing $\tilde{s}_{1}$ (or $\left.\tilde{s}_{5}\right)$ by one unit is $2 * c\left(c^{\prime}\right.$, respectively). Therefore, the relative cost of increasing the stock levels of product-specific components is higher in Problem-C than in Problem-NC, which 
implies that $\tilde{s}_{1}^{*}$ can be smaller than $s_{1}^{*}$. Interestingly, we also have $\tilde{s}_{5}^{*}<s_{3}^{*}+s_{4}^{*}$ in this example because $c \ll c^{\prime}$.

\section{Analysis under MFIFO}

In this section, we consider the same product structures as in $\S 3$ (see Figure 1) but under the MFIFO rule. All other assumptions and notations remain the same.

We first note that MFIFO belongs to a general class of reasonable allocation rules for System$\mathrm{C}$ that do not hold back stock when backorders exist. In particular, they satisfy the following condition:

$$
B^{i}(t) * \min \left\{I_{i}(t), I_{5}(t)\right\}=0, \quad i=1,2, \text { for all } t \geq 0,
$$

where $I_{i}(t)$ and $B^{j}(t)$ are the on-hand inventory of component $i$ and backorders of product $j$ at time $t$, respectively. Thus, under any rule that satisfies this property, a demand is backordered if and only if at least one of its components runs out of on-hand inventory. For this reason, we call rules that satisfy condition (10) no-holdback rules.

MFIFO is a no-holdback rule. Other no-holdback rules may allocate available inventory to backordered demand in different sequences from FIFO. For any no-holdback rule, we assume that demand for the same product is satisfied on a FIFO basis. In the next subsection, we shall show that, for any given base-stock policy, MFIFO achieves the same fill rates as any other no-holdback rule. Thus, under the framework of minimizing the total inventory investments subject to fill rate constraints, comparing MFIFO with FIFO is the same as comparing any no-holdback rule with FIFO.

\subsection{Fill Rate Expressions}

Proposition 5 Under any given base-stock policy in System-C,

1. the fill-rates under MFIFO can be expressed as follows,

$$
\begin{aligned}
& \tilde{f}^{1}=\operatorname{Pr}\left\{D^{1}\left(L_{1}\right)<\tilde{s}_{1}, D^{1}\left(L^{\prime}\right)+D^{2}\left(L^{\prime}\right)-\left[D^{2}\left(L_{2}\right)-\tilde{s}_{2}\right]^{+}<\tilde{s}_{5}\right\}, \\
& \tilde{f}^{2}=\operatorname{Pr}\left\{D^{2}\left(L_{2}\right)<\tilde{s}_{2}, D^{1}\left(L^{\prime}\right)+D^{2}\left(L^{\prime}\right)-\left[D^{1}\left(L_{1}\right)-\tilde{s}_{1}\right]^{+}<\tilde{s}_{5}\right\} .
\end{aligned}
$$

Note that $B_{2}=\left[D^{2}\left(L_{2}\right)-\tilde{s}_{2}\right]^{+}\left(\right.$or $\left.B_{1}=\left[D^{1}\left(L_{1}\right)-\tilde{s}_{1}\right]^{+}\right)$is the number of backorders of product-specific component 2 (or 1 ).

2. MFIFO always outperforms FIFO on the fill rates.

3. MFIFO has identical fill rates as any other no-holdback rule. 
Proof. See appendix for details.

In the rest of this section, we shall focus on MFIFO. By Proposition 5, we note that all results of MFIFO equally apply to no-holdback rules.

\subsection{P1-P3: Equal Lead Times}

In this subsection we assume the same system settings studied in $\S 3.1$. We show that, here, unlike the case under FIFO, properties P1 - P3 hold true under MFIFO. Hence, commonality cannot hurt.

First, we show that, under MFIFO, the optimal solution for System-NC achieves the same product fill rates in System-C, which implies P1. That is:

Proposition 6 Suppose $L_{i}=L^{\prime}$ for all $i$ and $c_{i}=c^{\prime}, i=3,4,5$. In addition, $L_{1}=L_{2}=L$ and $L^{\prime}=L$. Let $\tilde{s}_{i}=s_{i}^{*}, i=1,2$, and $\tilde{s}_{5}=s_{3}^{*}+s_{4}^{*}$. Then, under MFIFO,

$$
\tilde{f}^{j}=f^{j}, \quad j=1,2 .
$$

Hence, the solution $\underline{\tilde{s}}=\left(s_{1}^{*}, s_{2}^{*}, s_{3}^{*}+s_{4}^{*}\right)$ is feasible in System-C. Therefore, $\boldsymbol{P} \mathbf{1}$ holds.

Proof. See appendix for details.

For properties $\mathbf{P 2}$ and $\mathbf{P 3}$, we have

Proposition 7 Under MFIFO and equal lead times,

$$
\begin{aligned}
& \tilde{s}_{5}^{*} \leq s_{3}^{*}+s_{4}^{*}, \\
& \tilde{s}_{i}^{*} \geq s_{i}^{*}, i=1,2 .
\end{aligned}
$$

So, P2 and P3 hold even for non-identical $c_{i}, i=1,2$.

Proof. See appendix for details.

\subsection{P1-P3: Unequal Lead Times}

The main result in this subsection is the following observation:

Observation 8 When the lead times are not identical, P1-P3 may not hold under MFIFO.

When $\lambda^{j}, L_{i}, c_{i}$ are different for $j=1,2, i=1,2$, we have found counterexamples to $\mathbf{P} 1$ and P2. Table 3 presents one of them, with $\lambda^{1}=1, \lambda^{2}=2$.

In this example, using the optimal System-NC solution in System-C, i.e., assuming $\left(\tilde{s}_{1}, \tilde{s}_{2}, \tilde{s}_{5}\right)=$ $(11,8,46)$, we have $\left(\tilde{f}^{1}, \tilde{f}^{2}\right)=(89.99 \%, 94.69 \%)$, missing the target fill rate for product 1 . Because 
Table 3: A counterexample to $\mathbf{P 1}$ and $\mathbf{P 2}$ under MFIFO

\begin{tabular}{|c|c|c|c|c|c|c|c|}
\hline$\alpha$ & $\left(c_{1}, c_{2}\right.$, & $\left(L_{1}, L_{2}\right.$, & $\%$ & soln. (NC) & fill rates (NC) & soln. $(\mathrm{C})$ & fill rates $(\mathrm{C})$ \\
& $\left.c^{\prime}\right)$ & $\left.L^{\prime}\right)$ & Savings & $\left(s_{1}^{*}, s_{3}^{*}\right),\left(s_{2}^{*}, s_{4}^{*}\right)$ & $\left(f^{1}, f^{2}\right)$ & $\left(\tilde{s}_{1}^{*}, \tilde{s}_{2}^{*}, \tilde{s}_{5}^{*}\right)$ & $\left(\tilde{f}^{1}, \tilde{f}^{2}\right)$ \\
\hline $90 \%$ & $(10,10,1)$ & $(7,2,10)$ & $-0.42 \%$ & $(11,18),(8,28)$ & $(90.00 \%, 90.85 \%)$ & $(11,8,47)$ & $(90.06 \%, 94.77 \%)$ \\
\hline
\end{tabular}

the base-stock levels can only choose integers, making up the small deterioration can lead to sizable increase in inventory investment which results in higher inventory investment in System-C than in System-NC.

It turns out that the counterexample in $\S 3.2$ for P3 under FIFO (see Table 2) is also a counterexample for P3 under MFIFO, see Table 4. Here, the solution for System-C stays the same

Table 4: A counterexample to $\mathbf{P 3}$ under MFIFO

\begin{tabular}{|c|c|c|c|c|}
\hline$c / c^{\prime}$ & $\begin{array}{c}\text { soln. (NC) } \\
\left(s_{1}^{*}, s_{3}^{*}\right)\end{array}$ & fill rate $(\mathrm{NC})$ & $\begin{array}{c}\text { soln. }(\mathrm{C}) \\
\left(\tilde{s}_{1}^{*}, \tilde{s}_{5}^{*}\right)\end{array}$ & fill rate $(\mathrm{C})$ \\
\hline 0.1 & $(8,16)$ & $95.08 \%$ & $(6,29)$ & $95.22 \%$ \\
\hline
\end{tabular}

under both FIFO and MFIFO. The example also confirms the result in $\S 4.2$, i.e., with the same base-stock levels, MFIFO yields higher fill rates than FIFO (see Tables 2 and 4).

So far, we have provided some insight on when commonality always generates inventory benefits, and constructed counterexamples on when it does not. The counterexamples, although occurring infrequently and yielding minor losses, caution the use of this strategy in dynamic ATO systems. To provide a more comprehensive picture on the impact of component commonality, we conducted an asymptotic analysis and a numerical study in the following sections to quantify the value of commonality and its sensitivity.

\section{$5 \quad$ Asymptotic Analysis}

The counterexamples in the previous section indicate that if the common component is inexpensive relative to product-specific components, the value of commonality may be low. Thus, it is natural to ask: does it always pay to share expensive components in systems with lead times? More importantly, what is the impact of component cost, lead time and target fill rate on component commonality? We aim to address these questions in this section. 
To gain more insight, we consider a more general product structure than the one considered in the previous section. In particular, instead of two products, we now assume that there are $N$ products. Again, each product is made of two components, and we still use $i$ to index components and $j$ to index products. In the original system without component sharing, i.e, System-NC, product $j$ is assembled from two unique components $j$ and $N+j$. In a revised system, System-C, we replace components $N+1, N+2, \ldots, 2 N$ with common component $2 N+1$, so product $j$ is assembled from the product-specific component $j$ and the common component $2 N+1$.

All other features and notations of the model stay the same as in the two-product system in $\S 1$ and $\S 3$. As before, we assume that all components replaced by the common component have an identical unit cost of $c^{\prime}$ and an identical lead time of $L^{\prime}$, and that after component sharing, unit cost and lead time do not change. In other words, $c_{i}=c^{\prime}$ and $L_{i}=L^{\prime}, i=N+1, N+2, \ldots, 2 N+1$.

The mathematical programs for System-NC and System-C (the counterparts of (1) and (2)) are given as follows:

$$
\begin{aligned}
& \text { Problem-NC } \quad C^{*}=\min \quad \sum_{i=1}^{2 N} c_{i} s_{i} \\
& \text { s.t. } \quad f^{j}(\underline{s}) \geq \alpha^{j}, j=1,2, \ldots, N, \quad\left(s_{i}\right) \in \mathcal{Z}_{+}^{2 N} . \\
& \text { Problem-C } \quad \tilde{C}^{*}=\min \quad \sum_{i=1}^{N} c_{i} \tilde{s}_{i}+c^{\prime} \tilde{s}_{2 N+1} \\
& \text { s.t. } \quad \tilde{f}^{j}(\underline{\tilde{s}}) \geq \alpha^{j}, j=1,2, \ldots, N, \quad\left(\tilde{s}_{i}\right) \in \mathcal{Z}_{+}^{N+1} .
\end{aligned}
$$

where

$$
\begin{aligned}
& f^{j}(\underline{s})=\operatorname{Pr}\left\{D^{j}\left(L_{j}\right)<s_{j}, D^{j}\left(L^{\prime}\right)<s_{N+j}\right\}, \\
& \tilde{f}^{j}(\underline{\tilde{s}})=\operatorname{Pr}\left\{D^{j}\left(L_{j}\right)<\tilde{s}_{j}, \sum_{l=1}^{N} D^{l}\left(L^{\prime}\right)<\tilde{s}_{2 N+1}\right\}, \quad \text { under FIFO, } \\
& \tilde{f}^{j}(\underline{\tilde{s}})=\operatorname{Pr}\left\{D^{j}\left(L_{j}\right)<\tilde{s}_{j}, \sum_{l=1}^{N} D^{l}\left(L^{\prime}\right)-\sum_{l=1, \ldots, N ; l \neq j} B_{l}<\tilde{s}_{2 N+1}\right\}, \quad \text { under MFIFO. }
\end{aligned}
$$

Here, $B_{l}$ is the number of backorders of component $l$ in steady-state. Eqs. (18)-(19) are straightforward extensions of Eqs. (3)-(4). Eq. (20) is an extension of Eq. (11) of $N=2$, see appendix for a detailed discussion.

\subsection{Effect of Sharing Expensive Components}

Let us first consider the effect of sharing expensive components, such as using common CPUs in PCs and common air-conditioning systems in automobiles. We do so by assuming $c_{i} / c^{\prime} \rightarrow 0, i=$ 
$1,2, \ldots, N$. Under this condition, problem-NC (16), problem-C (17) reduce to,

$$
\begin{array}{rll}
\text { Problem-NC(0) } & \min & c^{\prime} \sum_{i=N+1}^{2 N} s_{i} \\
& \text { s.t. } & \operatorname{Pr}\left\{D^{j}\left(L^{\prime}\right)<s_{N+j}\right\} \geq \alpha^{j}, j=1,2, \ldots, N \\
\text { Problem-C(0) } & \min & c^{\prime} \tilde{s}_{2 N+1} \\
& \text { s.t. } & \operatorname{Pr}\left\{\sum_{l=1}^{N} D^{l}\left(L^{\prime}\right)<\tilde{s}_{2 N+1}\right\} \geq \alpha^{j}, j=1,2, \ldots, N
\end{array}
$$

This holds true because in the limit, we can increase base-stock levels of the product-specific components without increasing total inventory investments. This implies that for all productspecific components, we can always ensure $D^{i}\left(L_{i}\right)<s_{i}$ (and hence $B_{i}=0$ ), $i=1, \ldots, N$ without increasing objective function values. Therefore, the fill rates are determined only by components $i=N+1, \ldots, 2 N$ in System-NC and by common component $i=2 N+1$ in System-C. Note that Problem-C(0) has identical form under either FIFO or MFIFO.

To study the impact of component commonality, we follow standard procedure by assuming $\alpha^{j}=\alpha$ for all $j$, and approximating the Poisson lead time demand $D_{N+j}\left[t-L^{\prime}, t\right)$ by a normal random variable with mean $L^{\prime} \lambda^{j}$ and standard deviation $\sqrt{L^{\prime} \lambda^{j}}$. (This approximation is reasonably accurate when $L^{\prime} \lambda^{j}$ is not too small, e.g., $\left.L^{\prime} \lambda^{j} \geq 10\right)$. Let $\Phi(\cdot)$ be the standard normal distribution, and denote $z_{\alpha}=\Phi^{-1}(\alpha)$. Consistent with practice, we assume $\alpha>50 \%$ and so $z_{\alpha}>0$. Then for System-NC,

$$
s_{N+j}^{*}=L^{\prime} \lambda^{j}+z_{\alpha} \sqrt{L^{\prime} \lambda^{j}} .
$$

Thus, the optimal objective value of Problem-NC(0) equals $c^{\prime} L^{\prime} \sum_{j=1}^{N} \lambda^{j}+c^{\prime} z_{\alpha} \sqrt{L^{\prime}} \sum_{j=1}^{N} \sqrt{\lambda^{j}}$. Following the same logic, for Problem-C(0) we have,

$$
\tilde{s}_{2 N+1}^{*}=L^{\prime} \sum_{j=1}^{N} \lambda^{j}+z_{\alpha} \sqrt{L^{\prime}} \sqrt{\sum_{j=1}^{N} \lambda^{j}} .
$$

Hence, the optimal objective value of Problem-C(0) is $c^{\prime} \tilde{s}_{2 N+1}^{*}$. Note that both Eqs. (23)-(24) include two terms, the first term represents the pipeline inventory while the second term represents the safety-stock. The effect of lead time is predominately on pipeline inventory $\left(O\left(L^{\prime}\right)\right)$ rather than on safety-stock $\left(O\left(\sqrt{L^{\prime}}\right)\right)$.

By Eqs. (23)-(24),

Value of Component Commonality

$$
\equiv \text { Relative Reduction on Inventory Investment from System-NC to System-C }
$$




$$
=\frac{\sum_{j=1}^{N} \sqrt{\lambda^{j}}-\sqrt{\sum_{j=1}^{N} \lambda^{j}}}{\sqrt{L^{\prime}} \sum_{j=1}^{N} \lambda^{j} / z_{\alpha}+\sum_{j=1}^{N} \sqrt{\lambda^{j}}} \text { in case of } c_{i} / c^{\prime} \rightarrow 0, i=1,2, \ldots, N \text {. }
$$

When expressed in terms of percentage, we also refer to (25) as the percentage savings of component sharing. Eq. (26) shows that, in the special case of $c_{i}<<c^{\prime}$, the value of component commonality is always greater than or equal to zero. Note that including the pipeline inventory in Eq. (25) reduces the value of commonality, and makes it sensitive to the changes in lead times and fill rates.

To see clearly the impact of the system parameters, we consider the symmetric case of $\lambda^{j}=\lambda$, for all $j$. Under this setting, (26) is simplified to

$$
\left(\frac{z_{\alpha}}{z_{\alpha}+\sqrt{\lambda L^{\prime}}}\right)\left(1-\frac{1}{\sqrt{N}}\right)
$$

Thus, when $c_{i}<<c^{\prime}$, the value of component commonality increases as (1) the target service level $\alpha$ increases, (2) the lead time for the common component $L^{\prime}$ decreases, and (3) the number of products that share the common component $N$ increases, at a rate proportional to the square root of $N$.

In addition, in the case of symmetric products, the total stock level of the common component in System-C (under either FIFO or MFIFO) is $N L^{\prime} \lambda+z_{\alpha} \sqrt{N L^{\prime} \lambda}$. This resembles the "risk pooling" effect studied by Eppen (1979) for centralized stock. Indeed, Problem-NC(0) can be viewed as a completely decentralized multi-location system with separate inventory kept at each location in order to satisfy local demand; Problem-C(0) can be viewed as a completely centralized system with one central warehouse fulfilling all demands. (The "risk pooling" effect refers to the observation that expected inventory-related costs in a decentralized system exceed those in a centralized system, and when demands are identical and uncorrelated, the part of cost proportional to $z_{\alpha} \sqrt{N L^{\prime} \lambda}$ in a centralized system increases as the square root of the number of consolidated demands).

\subsection{Effect of Sharing Inexpensive Components}

Let us next consider the effect of sharing inexpensive components, such as using common mouse in PCs and common spark plugs in automobiles. We do so by assuming $c^{\prime} / c_{i} \rightarrow 0$ for $i=1,2, \ldots, N$. Under this condition, problem-NC (16) reduces to,

$$
\begin{array}{lll}
\text { Problem-NC }(\infty) \quad \min & \sum_{i=1}^{N} c_{i} s_{i} \\
\text { s.t. } & \operatorname{Pr}\left\{D^{j}\left(L_{j}\right)<s_{j}\right\} \geq \alpha^{j}, j=1,2, \ldots, N,
\end{array}
$$


Problem-C (17) (under either FIFO or MFIFO) reduces to,

$$
\begin{array}{lll}
\text { Problem-C }(\infty) \quad \min & \sum_{i=1}^{N} c_{i} \tilde{s}_{i} \\
\text { s.t. } & \operatorname{Pr}\left\{D^{j}\left(L_{j}\right)<\tilde{s}_{j}\right\} \geq \alpha^{j}, j=1,2, \ldots, N .
\end{array}
$$

Observe that the mathematical programs (28) and (29) are identical, hence the value of component commonality converges to zero as $c^{\prime} / c_{i} \rightarrow 0, i=1,2, \ldots, N$.

To summarize, we have shown the following results.

Proposition 9 In the multi-product System-NC and System- $C$, the following results hold under either FIFO or MFIFO:

1. Given $c^{\prime}$, the value of component commonality converges to that of the "risk pooling" effect as $c_{i} / c^{\prime} \rightarrow 0$ for all $i=1,2, \ldots, N$.

2. Given $c_{i}, i=1,2, \ldots, N$, the value of component commonality converges to zero as $c^{\prime} / c_{i} \rightarrow 0$ for all $i=1,2, \ldots, N$.

Proposition 9 implies that, under general conditions, if the common component is very expensive relative to product-specific components, then the impact of component commonality is substantial. In fact, the value of component commonality converges to that of the "risk pooling" effect. On the other hand, if the common component is very cheap relative to all the product specific components, component commonality has little impact on inventory investments and service levels.

\section{$6 \quad$ Numerical Studies}

The previous section quantifies the value of commonality in the limiting cases of component costs. In this section, we conduct a numerical study to quantify the value of component commonality in between the two asymptotic limits. We are interested in the impact of component costs and lead times, and target fill rates. We also quantify the performance gap between FIFO and MFIFO rules. We shall connect results in this section to those in previous sections whenever possible.

To develop managerial insights, we focus on the simple two-product system (Figure 1) under the symmetric assumption: $\lambda^{j}=\lambda$ and $\alpha^{j}=\alpha$ for $j=1,2 ; L_{i}=L$ and $c_{i}=c$ for $i=1,2$. Note that $c \neq c^{\prime}$ and $L \neq L^{\prime}$ in general. Clearly, we must have identical $s_{i}^{*}$ and $\tilde{s}_{i}^{*}$ across $i=1,2$, and identical $s_{i}^{*}$ for $i=3,4$ under either FIFO or MFIFO.

The mathematical programs (16) and (17) are solved by enumeration based on the exact evaluation of the fill rates (see Eqs. (18)-(20)). 


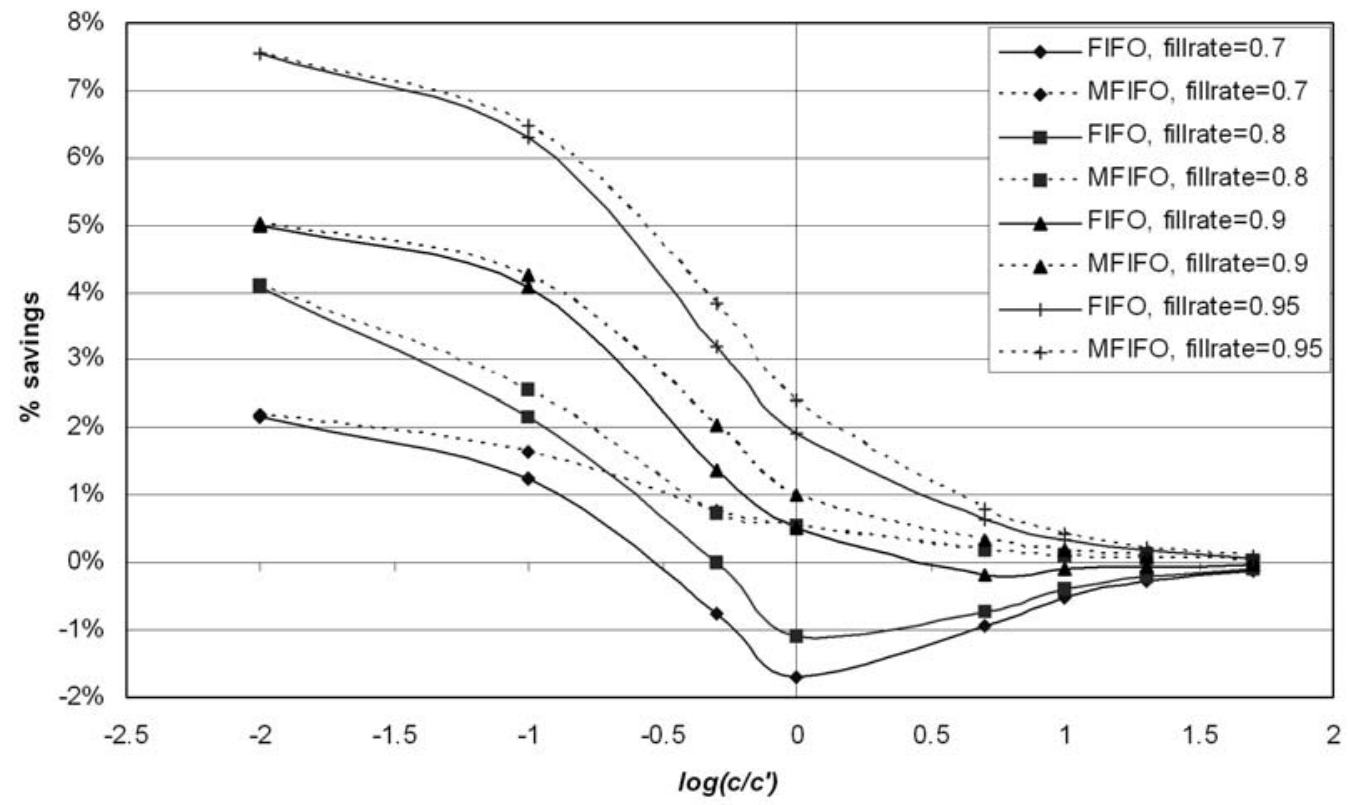

Figure 2: The impact of component costs and target fill rate. $L^{\prime}=L=20, \lambda=2$.

\subsection{Parameter Setup}

The system under study has the following parameters: $\lambda, c / c^{\prime}, L^{\prime}, L / L^{\prime}$ and $\alpha$. We choose the following values for each parameter. $\lambda \in\{1,2,5\} ; c / c^{\prime} \in\{0.01,0.1,0.5,1,5,10,20,30\} ; L^{\prime} \in$ $\{1,2,5,10,20\} ; L / L^{\prime} \in\{0.1,0.2,0.5,1,2,3\} ;$ and finally $\alpha \in\{0.7,0.75,0.8,0.85,0.9,0.95\}$. Given a combination of the parameters, we compute the value of commonality under either FIFO or MFIFO.

Because the base-stock levels can only choose integers, the target fill rate can rarely be achieved exactly. In fact, the difference between the target fill rate and the actual fill rate can be high, e.g., up to $9 \%$ at $\lambda * L^{\prime}=1$. As $\lambda * L^{\prime}$ increases, the integer base-stock level effect diminishes.

\subsection{Effect of Component Costs and Target Fill Rates}

To study the impact of component cost ratios $\left(c / c^{\prime}\right)$ and target fill rate $(\alpha)$ on the value of commonality, we consider the equal lead time case $L / L^{\prime}=1$. We fix the values of $L^{\prime}$ and $\lambda$, but vary $c / c^{\prime}$ and $\alpha$. Figure 2 shows a representative case with $L^{\prime}=L=20$ and $\lambda=2$.

We observe the following:

- When $c \ll c^{\prime}$ (so $\left.\log \left(c / c^{\prime}\right) \ll 0\right)$, the value of component commonality can be substantial. Specifically, Figure 2 shows that the limiting values are $2.2 \%, 4.1 \%, 5 \%, 7.5 \%$ for $\alpha=0.7,0.8,0.9,0.95$ respectively (under FIFO), which are reasonably close to $2.2 \%, 3.4 \%$, 
$4.9 \%, 6.1 \%$ given by the asymptotic analysis (Eq. 26). The differences are due to integer base-stock levels. As predicted by the asymptotic results, the value of commonality under MFIFO converges to the same limits as that under FIFO.

- When $c \gg c^{\prime}\left(\right.$ so $\left.\log \left(c / c^{\prime}\right) \gg 0\right)$, the value of component commonality always converges to zero. This observation also confirms the asymptotic result.

- When $c / c^{\prime}$ is in the middle range, the value of component commonality tends to decrease as $c / c^{\prime}$ increases for all $\alpha$ and under either FIFO or MFIFO. However, the value of commonality is not always monotonic with respect to $c / c^{\prime}$ due to the integer base-stock level effect. As shown in Proposition 5, MFIFO always outperforms FIFO on the value of commonality. Furthermore, while commonality may not generate benefits under FIFO, commonality is always beneficial under MFIFO.

- The value of commonality tends to increase as the target fill rate increases.

- The gap between FIFO and MFIFO (i.e., the difference in the value of commonality) can be comparable to the value of commonality, and the gap tends to increase as fill rate decreases.

These observations remain the same for other $\lambda, L^{\prime}$ and $L / L^{\prime}$ tested.

To further quantify the impact of fill rate on the performance gap between FIFO and MFIFO, we compute, for each $\alpha$, the value of commonality under either FIFO or MFIFO for all combinations of $L^{\prime}, L / L^{\prime}, c / c^{\prime}$ and $\lambda$. The result is summarized in Table 5 which confirms our observation from Figure 2. That is, as the target fill rate, $\alpha$, decreases, the performance gap tends to increase, and therefore, the advantage of MFIFO over FIFO tends to increase.

Table 5: The impact of target fill rate on performance gap between FIFO and MFIFO

\begin{tabular}{|c|c|c|c|c|c|c|}
\hline$\alpha$ & 0.7 & 0.75 & 0.8 & 0.85 & 0.9 & 0.95 \\
\hline Mean gap & $0.41 \%$ & $0.51 \%$ & $0.20 \%$ & $0.23 \%$ & $0.15 \%$ & $0.09 \%$ \\
\hline Max gap & $16.67 \%$ & $9.09 \%$ & $5.56 \%$ & $6.25 \%$ & $11.11 \%$ & $2.63 \%$ \\
\hline
\end{tabular}

To explain this observation, we rewrite the fill rate expressions for FIFO and MFIFO as follows,

$$
\begin{aligned}
& \tilde{f}^{1}=\operatorname{Pr}\left\{D^{1}\left(L_{1}\right)<\tilde{s}_{1}, D^{1}\left(L^{\prime}\right)+D^{2}\left(L^{\prime}\right)<\tilde{s}_{5}\right\} \quad \text { under FIFO } \\
& \tilde{f}^{1}=\operatorname{Pr}\left\{D^{1}\left(L_{1}\right)<\tilde{s}_{1}, D^{1}\left(L^{\prime}\right)+D^{2}\left(L^{\prime}\right)-\left[D^{2}\left(L_{2}\right)-\tilde{s}_{2}\right]^{+}<\tilde{s}_{5}\right\} \text { under MFIFO, }
\end{aligned}
$$




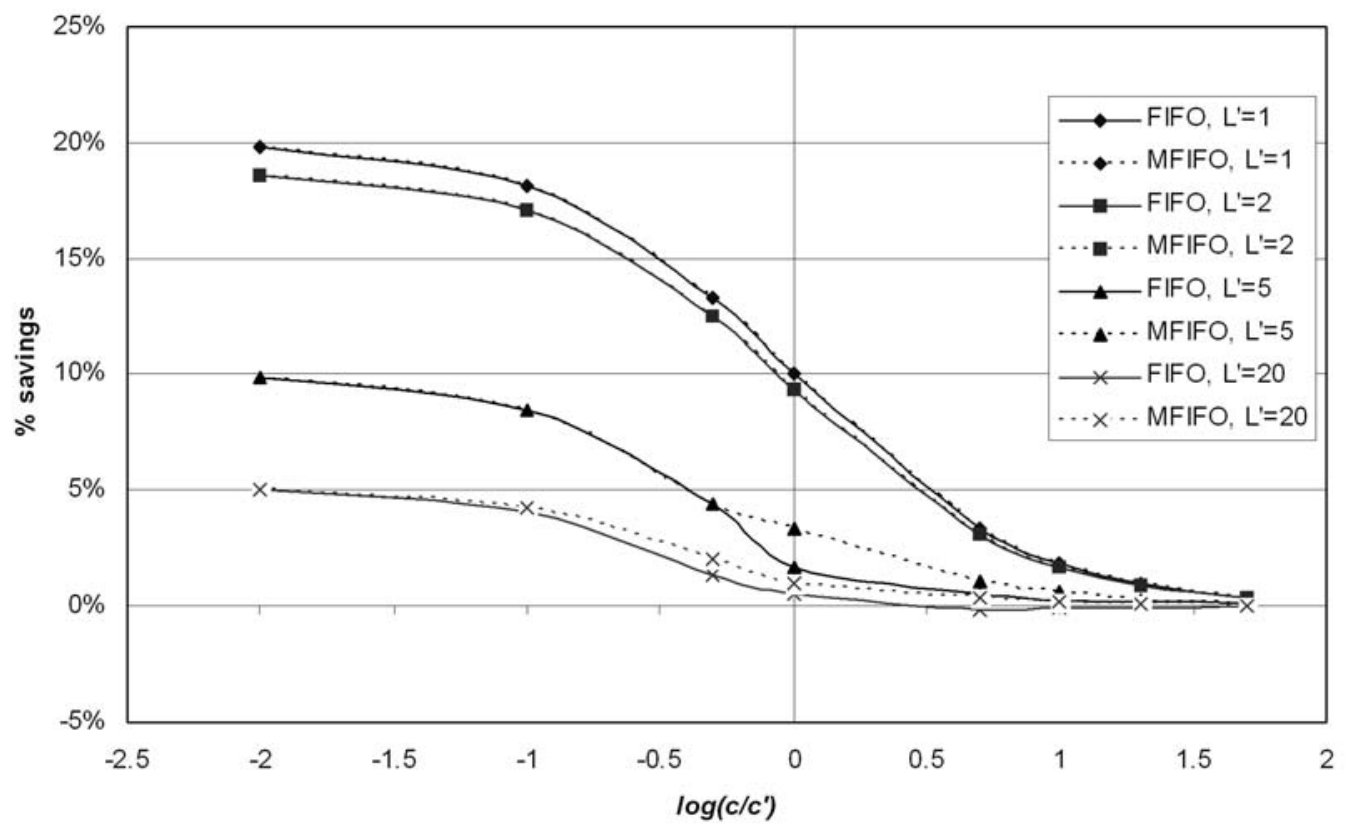

Figure 3: The impact of component costs and common component lead time. $\alpha=0.9, \lambda=2$ and $L^{\prime}=L$.

where $B_{2}=\left[D^{2}\left(L_{2}\right)-\tilde{s}_{2}\right]^{+}$is the backorder of product-specific component 2. Note that the fill rates differ only by $B_{2}$ which tends to increase at a lower target fill rate, thus the performance gap between FIFO and MFIFO tends to be larger as the target fill rate decreases.

The parameter combinations corresponding the maximum gaps share some common features. Mostly interestingly, $c / c^{\prime}$ equals to either 1 or 0.5 in all cases. This is intuitive because by the asymptotic result, the value of commonality converges to the same value under either FIFO or MFIFO in the limits of $c / c^{\prime}$. So the maximum performance gap between FIFO and MFIFO is most likely to occur when $c / c^{\prime}$ is equally far away from the limits.

\subsection{Effect of Component Lead Times}

In $\S 5$, we discuss the impact of the lead time of the common component $L^{\prime}$, in the limiting cases of $c / c^{\prime}$. We now study this impact when $c / c^{\prime}$ is in the middle range. We again consider equal lead times, i.e., $L^{\prime}=L$. We fix $\alpha$ and $\lambda$ but vary $c / c^{\prime}$ and $L^{\prime}$. Figure 3 provides a representative case with $\alpha=0.9$ and $\lambda=2$.

We make the following observations:

- The value of commonality tends to decrease as $L^{\prime}$ increases under either FIFO or MFIFO.

- The gap between MFIFO and FIFO does not seem to depend on $L^{\prime}$. 


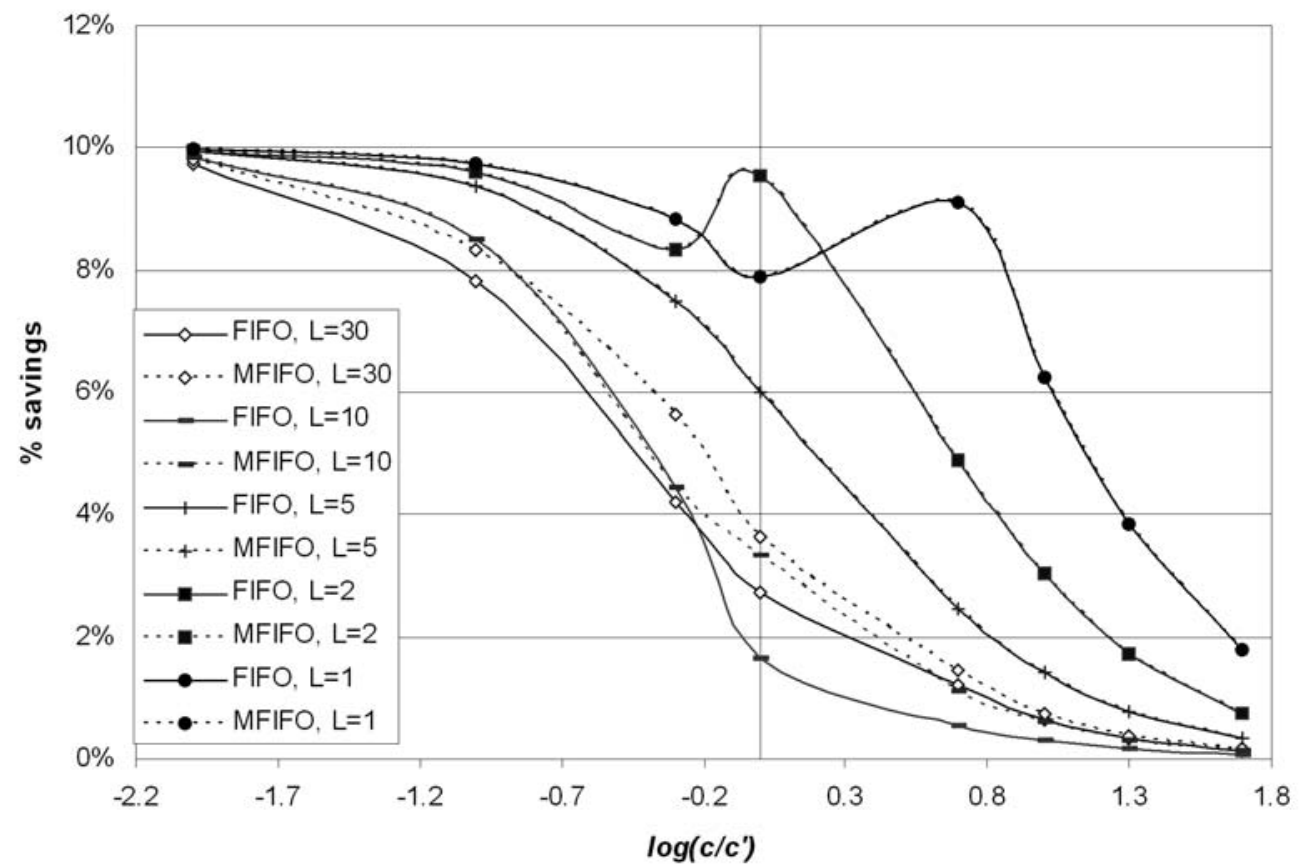

Figure 4: The impact of component costs and product-specific component lead times. $\alpha=0.9$, $\lambda=1$ and $L^{\prime}=10$.

The observations remain the same for other $\lambda, \alpha$ and $L / L^{\prime}$.

We next study the impact of product-specific component lead time $(L)$ on the value of commonality. We fix $\alpha, \lambda$ and $L^{\prime}$, but vary $c / c^{\prime}$ and $L$. Figure 4 shows the results for $\alpha=0.9, \lambda=1$ and $L^{\prime}=10$. The impact of $L$ is more complex than that of $L^{\prime}$ under either FIFO or MFIFO, as summarized below:

- $L$ can have a significant impact on the value of component commonality. The impact is greater when $c / c^{\prime}$ is in between the asymptotic limits. Consistent with the asymptotic analysis, the value of component commonality converges to the same limit as $c / c^{\prime}$ approaches zero, regardless of the lead times of the product-specific components.

- In general, the value of commonality is greater when $L<L^{\prime}$ than when $L \geq L^{\prime}$. To explain this observation intuitively, let's consider the extreme case of $L / L^{\prime} \ll 1$. Here, because the product-specific components have negligible lead times, one can approximate the system performance by assuming immediate availability of these components. Doing so effectively removes the product-specific components from the system, and therefore the value of commonality approaches the "risk pooling" effect.

- When $L<L^{\prime}$, the value of component commonality tends to increase as $L$ decreases. That 
is, if the lead time of the common component is longer than those of the product-specific components, the value of component commonality tends to increase as the lead time difference increases. We should point out that there are exceptions to this trend due to the discrete base-stock levels. When $L \geq L^{\prime}$, the trend is not clear.

These observations are supported by numerical studies of other parameter sets with different $\alpha, \lambda$ and $L^{\prime}$ (not reported here).

Finally, we study the impact of $L / L^{\prime}$ on the performance gap between FIFO and MFIFO. To this end, we compute, for each $L / L^{\prime}$, all combinations of $L^{\prime}, \alpha, c / c^{\prime}$, and $\lambda$. Table 6 summarizes the results. The table shows that at $L / L^{\prime} \leq 1$, the gap clearly depends on $L / L^{\prime}$. Specifically, as $L / L^{\prime}$ decreases, the gap tends to decrease. Intuitively, this is true because by Eq. (11), if $L / L^{\prime}<1$ and continues decreasing, the backorder of the product-specific components becomes less significant as compared to the lead time demand for the common component. Thus, the fill rate difference between FIFO and MFIFO becomes smaller. However, at $L / L^{\prime}>1$, the trend is not clear.

Table 6: The impact of $L / L^{\prime}$ on performance gap between FIFO and MFIFO

\begin{tabular}{|c|c|c|c|c|c|c|}
\hline$L / L^{\prime}$ & 3 & 2 & 1 & 0.5 & 0.2 & 0.1 \\
\hline Mean gap & $0.27 \%$ & $0.26 \%$ & $0.58 \%$ & $0.31 \%$ & $0.15 \%$ & $0.01 \%$ \\
\hline Max gap & $9.09 \%$ & $6.67 \%$ & $16.67 \%$ & $7.14 \%$ & $5.56 \%$ & $1.35 \%$ \\
\hline
\end{tabular}

The parameter combinations corresponding to the maximum gaps here also share some common features. In addition to the fact that $c / c^{\prime}$ equals to either 1 or 0.5 in all cases, the target fill rates are no more than $80 \%$, which confirms the result of Table 5 .

\section{Concluding Remarks}

We have studied the value of component commonalty and its sensitivity to system parameters in a class of dynamic ATO systems with lead times. Since the optimal policy is not known, we have assumed that component inventories are replenished by independent base-stock policies and are allocated according to either the FIFO or the MFIFO rule. These policies are widely used in practice.

Our analysis shows that while component commonality is generally beneficial, its value depends strongly on the component costs, lead times, and allocation rules (FIFO or MFIFO). In particular, commonality does not guarantee inventory benefits for certain systems, e.g., those with identical 
lead times under FIFO, and those with non-identical lead times under either FIFO or MFIFO. On the other hand, with identical lead times and MFIFO, component commonality always yields inventory benefits. We also show that MFIFO always outperforms FIFO on the value of commonality, and MFIFO achieves the same product fill rates as any other no-hold-back rule. Using asymptotic analysis and a numerical study, we quantify the impact of component costs and lead times, and target fill rates on the value of commonality and the performance gap between MFIFO and FIFO.

It is worth mentioning that our objective function - the total investment in the base-stock levels - is an approximation of the component inventory holding costs. This choice is common in the inventory literature. It also facilitates the comparison with the static model. However, this approximation does not account for the extra holding costs that are charged in the FIFO system on the components that are reserved when the matching components are unavailable. This fact affects the economics of the FIFO allocation rule adversely, and thus further supports our results on the comparison between FIFO and MFIFO.

Our findings, such as those of Proposition 9, are consistent with many industry practices, but not all. For example, in the medical equipment industry, a product family is often developed around a major component with long lead time (de Kok 2003). In the electronics industry, different cell phone models, e.g., Motorola, can share a platform (Whyte 2003), which is defined to be a set of key (and therefore more expensive) components and sub-system assets (Krishnan and Gupta 2001). In many other industries, however, both low and high-value components are shared among different product models. For computers, for example, the low-value common components include keyboard and mouse, and high-value common components include CPUs and hard disks. For automobiles, a low-value common component example is a spark plug, and a high-value common component example is a air-conditioning system. This phenomenon may reflect the fact that standardized components simplify procurement and exploit economies of scale in production, quality control and logistics. Those features are not included in our model.

For ease of exposition and tractability, we have focused on the continuous-time base-stock policy for inventory replenishment. We note that, in practice, the batch-ordering policy is very common. Under the batch-ordering policy, it is not clear whether Propositions 1 and 6 still hold. But clearly, Proposition 9 can be extended to handle batch orders. We also believe that counterexamples can be constructed under either FIFO or MFIFO because the fill rates of a batch ordering system can be expressed as the averages of their counterparts in the base-stock systems (Song 2000).

A dynamic model with lead time differs from a static model without lead time because in the 
former, both demand and replenishment arrive at the system gradually, while in the latter, all demand and inventory present at the same time. Thus, in the dynamic model, lead times and the allocation rule that dynamically assigns common components to demand realized to date play important roles in realizing the value of component commonality. As the present work neared completion, we learned the recently completed work by Dogru, Reiman and Wang (2007). They established a lower bound for System-C with equal constant lead times under the backorder-cost model (as opposed to the service-level constrained model considered in our paper). They showed that MFIFO is the optimal allocation rule under certain symmetric conditions. However, when the symmetric condition is violated or lead times are not identical, or when a service-level constrained model is used, the form of the optimal allocation rule remains open for future research.

Acknowledgments. We thank the Editor-in-Chief, Professor Gerard Cachon, the Associate Editor and three anonymous referees for their constructive comments and thoughtful suggestions. We also thank Paul Zipkin and seminar attendants at Duke University and Cornell University for helpful comments on an earlier version of this paper. The first author was supported in part by Award No. 70328001 from the National Natural Science Foundation of China. The second author was supported in part by a Faculty Research Grant from Rutgers Business School-Newark and New Brunswick, and by a CAREER Award No. 0747779 from the National Science Foundation.

\section{Appendix}

Proof of Observation 2. First note that the objective function of Problem-NC is a monotonically increasing function of the stock levels, thus $s_{i}^{*}, i=1,2,3,4$ must be the smallest integers such that the fill rate constraint in (1) is satisfied. By Eq. (9),

$$
\operatorname{Pr}\left\{D^{j}(L)<s_{j}^{*}-1\right\}<\alpha^{j} \leq \operatorname{Pr}\left\{D^{j}(L)<s_{j}^{*}\right\}, j=1,2 .
$$

By Eq. (4), the optimal solution of System-C satisfies $\operatorname{Pr}\left\{D^{j}(L)<\tilde{s}_{j}^{*}, D^{1}(L)+D^{2}(L)<\tilde{s}_{5}^{*}\right\} \geq \alpha^{j}$. For this fill rate constraint to hold, it follows from inequality (30) that $\tilde{s}_{i}^{*} \geq s_{i}^{*}, i=1,2$, and therefore P3 holds.

Proof of Proposition 5. We derive the product fill rate expressions for System-C under any noholdback rule which includes MFIFO as a special case. For any $u \geq 0$, let $D_{i}(t, t+u]$ and $D^{j}(t, t+u]$ be the total demand for component $i$ and product $j$ during interval $(t, t+u]$, respectively. Then $D_{i}(t, t+u]=D^{i}(t, t+u], i=1,2$, and $D_{5}(t, t+u]=D^{1}(t, t+u]+D^{2}(t, t+u]$. Let $I N_{i}(t)$ be the 
net inventory under FIFO. Then,

$$
I N_{i}(t)=\tilde{s}_{i}-D_{i}\left(t, t+L_{i}\right]
$$

and $B_{i}(t)=\left[-I N_{i}(t)\right]^{+}$is the number of backorders of component $i$ at time $t$ under FIFO.

Let $A_{i}^{j}(t)$ be the number of component $i$ available at time $t$ for fulfilling product $j$. Since the product-specific component $i$ is used only for product $i$, we can assume the FIFO rule for this component without changing system performance. Hence, all previous demands of product $i$ must be satisfied before any future product $i$ demand can be filled. Consequently, we have

$$
A_{i}^{i}(t)=\left[I N_{i}(t)\right]^{+}, \quad i=1,2 .
$$

Now, consider $A_{5}^{1}(t)$. By definition, $\left[I N_{5}(t)\right]^{+}$is the excessive inventory of component 5 at time $t$ after subtracting all demands of products 1 and 2 by time $t$. Note that some of those product-2 demands have been filled by time $t$, but some haven't. Those that haven't constitute $B^{2}(t)$, the total number of product-2 backorders at $t$. Because we have already subtracted these $\left(B^{2}(t)\right)$ demands from component 5's inventory in obtaining $I N_{5}(t)$, but these units should be made available for fulfilling product 1 demand due to condition (10), so we have

$$
A_{5}^{1}(t)=\left[I N_{5}(t)+B^{2}(t)\right]^{+} .
$$

Note that $B^{1}(t)$ shouldn't be included in the brackets of the above equation because demand of product 1 is satisfied on a FIFO basis.

Recall that a demand enters the backorder list if and only if at least one component required is not in stock at the time of its arrival (condition 10). In particular, among $B^{2}(t)$, there are $B_{2}(t)$ of them that are due to at least the shortage of component 2. If $B^{2}(t)=B_{2}(t)$, then

$$
A_{5}^{1}(t)=\left[I N_{5}(t)+B_{2}(t)\right]^{+} .
$$

If $B^{2}(t)>B_{2}(t)$, then there exist product-2 backorders that are due to the shortage of component 5 only, so we must have

$$
A_{5}^{1}(t)=0
$$

Because $A_{5}^{1}(t)=\left[I N_{5}(t)+B^{2}(t)\right]^{+}$and $B^{2}(t)>B_{2}(t)$, we must have $I N_{5}(t)<0$ and $A_{5}^{1}(t)=$ $\left[I N_{5}(t)+B_{2}(t)\right]^{+}$in this case. So, in either case,

$$
A_{5}^{1}(t)=\left[I N_{5}(t)+B_{2}(t)\right]^{+} .
$$


By the same logic,

$$
A_{5}^{2}(t)=\left[I N_{5}(t)+B_{1}(t)\right]^{+}
$$

Observe that, for each $i, D_{i}\left(t, t+L_{i}\right]$ has steady-state limit $D_{i}\left(L_{i}\right)$, which is the lead time demand following a Poisson random variable with mean $\lambda_{i} L_{i}$. Similarly, for each $j, D^{j}\left(t, t+L_{i}\right]$ has steady-state limit $D^{j}\left(L_{i}\right)$, which is a Poisson random variable with mean $\lambda^{j} L_{i}$. Moreover, $D^{1}\left(L_{i}\right)$ and $D^{2}\left(L_{i}\right)$ are independent of each other, and $D_{5}\left(L^{\prime}\right)=D^{1}\left(L^{\prime}\right)+D^{2}\left(L^{\prime}\right)$. Consequently, the stochastic processes $I N_{i}(t), B_{i}(t)$, and $A_{i}^{j}(t)$ defined above have steady-state limits. Denote these limits by $I N_{i}, B_{i}$ and $A_{i}^{i}$, respectively. Then,

$$
I N_{i}=\tilde{s}_{i}-D_{i}\left(L_{i}\right), \quad B_{i}=\left[D_{i}\left(L_{i}\right)-\tilde{s}_{i}\right]^{+}
$$

and

$$
A_{5}^{1}=\left[I N_{5}+B_{2}\right]^{+}, \quad A_{5}^{2}=\left[I N_{5}+B_{1}\right]^{+} .
$$

By PASTA (Poisson Arrivals See Time Averages) and Eqs. (5)-(6), we have

$$
\begin{aligned}
\tilde{f}^{1} & =\operatorname{Pr}\left\{A_{1}^{1}>0, A_{5}^{1}>0\right\} \\
& =\operatorname{Pr}\left\{I N_{1}>0, I N_{5}+B_{2}>0\right\} \\
& =\operatorname{Pr}\left\{D^{1}\left(L_{1}\right)<\tilde{s}_{1}, D^{1}\left(L^{\prime}\right)+D^{2}\left(L^{\prime}\right)-\left[D^{2}\left(L_{2}\right)-\tilde{s}_{2}\right]^{+}<\tilde{s}_{5}\right\} \\
\tilde{f}^{2} & =\operatorname{Pr}\left\{A_{2}^{2}>0, A_{5}^{2}>0\right\} \\
& =\operatorname{Pr}\left\{I N_{2}>0, I N_{5}+B_{1}>0\right\} \\
& =\operatorname{Pr}\left\{D^{2}\left(L_{2}\right)<\tilde{s}_{2}, D^{1}\left(L^{\prime}\right)+D^{2}\left(L^{\prime}\right)-\left[D^{1}\left(L_{1}\right)-\tilde{s}_{1}\right]^{+}<\tilde{s}_{5}\right\} .
\end{aligned}
$$

Comparing Eqs. (11)-(12) with Eq. (9), and noting that MFIFO also satisfies condition (10), the proof is complete for all three Claims.

Proof of Proposition 6. Because of equal lead times, the optimal base-stock levels in System-NC must satisfy $s_{1}^{*}=s_{3}^{*}$ and $s_{2}^{*}=s_{4}^{*}$. Then, product-1 fill rate in System-NC is

$$
f^{1}=\operatorname{Pr}\left\{D^{1}(L)<s_{1}^{*}, D^{1}(L)<s_{3}^{*}\right\}=\operatorname{Pr}\left\{D^{1}(L)<s_{1}^{*}\right\}
$$

Because $\tilde{s}_{5}=s_{3}^{*}+s_{4}^{*}=s_{1}^{*}+s_{2}^{*}$, product-1 fill rate in System-C satisfies

$$
\begin{aligned}
\tilde{f}^{1} & =\operatorname{Pr}\left\{D^{1}(L)<s_{1}^{*}, D^{1}(L)+D^{2}(L)-\left[D^{2}(L)-s_{2}^{*}\right]^{+}<s_{1}^{*}+s_{2}^{*}\right\} \\
& =\operatorname{Pr}\left\{D^{1}(L)<s_{1}^{*}, D^{1}(L)<s_{1}^{*}+\left[s_{2}^{*}-D^{2}(L)\right]^{+}\right\} \\
& =\operatorname{Pr}\left\{D^{1}(L)<s_{1}^{*}\right\} .
\end{aligned}
$$


Thus, $f^{1}=\tilde{f}^{1}$. A similar argument leads to $f^{2}=\tilde{f}^{2}$.

Proof of Proposition 7. We first prove inequalities (15). Note that the fill rate

$$
\tilde{f}^{1}=\operatorname{Pr}\left\{D^{1}(L)<\tilde{s}_{1}, D^{1}(L)+D^{2}(L)-\left[D^{2}(L)-\tilde{s}_{2}\right]^{+}<\tilde{s}_{5}\right\}
$$

is no larger than the marginal probability $\operatorname{Pr}\left\{D^{1}(L)<\tilde{s}_{1}\right\}$. Also, $\operatorname{Pr}\left\{D^{1}(L)<s_{1}^{*}-1\right\}<\alpha^{1} \leq$ $\operatorname{Pr}\left\{D^{1}(L)<s_{1}^{*}\right\}$ (see Eq. (30)). Therefore, we must have $\tilde{s}_{1}^{*} \geq s_{1}^{*}$ in order for $\tilde{f}^{1} \geq \alpha^{1}$. The same analysis applies to product-2.

To prove inequality (14), we note that, from Proposition $6, \tilde{f}^{j}=f^{j}$ when $\tilde{s}_{i}=s_{i}^{*}, i=1,2$ and $\tilde{s}_{j}=s_{3}^{*}+s_{4}^{*}, j=1,2$. Because $\tilde{s}_{i}^{*} \geq s_{i}^{*}, i=1,2$ (by Eq. (15)), the smallest $\tilde{s}_{5}$ for $\tilde{f}^{j}(t) \geq \alpha^{j}$ must be no larger than $s_{3}^{*}+s_{4}^{*}$.

To provide justifications for Eq. (20), we let $B^{i}$ be the steady-state product- $i$ backorders. Using the same logic as for $N=2$, we must have $B^{i} \geq B_{i}$ for $i=1, \ldots, N$, and

$$
A_{2 N+1}^{1}(t)=\left[I N_{2 N+1}(t)+\sum_{i=2}^{N} B^{i}\right]^{+} .
$$

If $B^{i}=B_{i}$ for all $i=2, \ldots, N$, then $A_{2 N+1}^{1}(t)=\left[I N_{2 N+1}(t)+\sum_{i=2}^{N} B_{i}\right]^{+}$. Otherwise, there must exist at least one product-specific component $l$ such that $B^{l}>B_{l}$. This implies that $A_{2 N+1}^{1}(t)=0$. Because $B^{i} \geq B_{i}$ for $i=1, \ldots, N$, we can write $A_{2 N+1}^{1}(t)=\left[I N_{2 N+1}(t)+\sum_{i=2}^{N} B_{i}\right]^{+}$in this case. So in either case,

$$
A_{2 N+1}^{1}(t)=\left[I N_{2 N+1}(t)+\sum_{i=2}^{N} B_{i}\right]^{+} .
$$

The rest of justification follows the discussion of $N=2$.

\section{References}

[1] Agrawal, N. \& M. Cohen (2001). Optimal material control and performance evaluation in an assembly environment with component commonality. Naval Research Logistics, 48, 409-429.

[2] Akcay, Y. \& S. H. Xu (2004). Joint inventory replenishment and component allocation optimization in an assemble-to-order system. Management Science, 50, 99-116.

[3] Alfaro, J. \& C. Corbett (2003). The value of SKU rationalization in practice (The pooling effect under suboptimal policies and nonnormal demand). Production and Operations Management, 12, 12-29. 
[4] Bagchi, U. \& G. Gutierrez (1992). Effect of increasing component commonality on service level and holding cost. Naval Research Logistics, 39, 815-832.

[5] Baker, K. R. (1985). Safety stock and component commonality. Journal of Operations Management, 6, 13-22.

[6] Baker, K. R., M. J. Magazine \& H. L. W. Nuttle (1986). The effect of commonality on safety stocks in a simple inventory model. Management Science, 32, 982-988.

[7] Benjaafar, S. \& M. ElHafsi (2006). Production and inventory control of a single product Assemble-to-Order system with multiple customer classes. Management Science, 52, 18961912.

[8] Chopra, S. \& P. Meindl (2001). Supply Chain Management. Prentice-Hall, NJ.

[9] Cheng, F., M. Ettl, G. Y. Lin \& D. D. Yao (2002). Inventory-service optimization in configureto-order systems. Manufacturing and Service Operations Management, 4, 114-132.

[10] Collier, D. A. (1981). The measurement and operating benefits of component part commonality. Decision Sciences, 12, 85-96.

[11] Collier, A. A. (1982). Aggregate safety stock levels and component part commonality. Management Science, 28, 1296-1303.

[12] Dogru, M., M. Reiman, Q. Wang (2007). A stochastic programming based inventory policy for assemble-to-order systems with application to the $\mathrm{W}$ model. Working paper, Alcatel-Lucent Bell Labs. NJ.

[13] De Kok, Ton G. (2003). Evaluation and optimization of strongly ideal Assemble-To-Order systems. Working Paper, Technische Universiteit Eindhoven. The Netherlands.

[14] Desai, P., S. Kekre, S. Radhakrishnan \& K. Krinivasan (2001). Product differentiation and commonality in design: Balancing revenue and cost drivers. Management Science, 47, 37-51.

[15] Deshpande, V., M. A. Cohen \& K. Donohue (2003). A threshold inventory rationing policy for service-differentiated demand classes. Management Science, 49, 683-703.

[16] Eppen, G. D. (1979). Effects of centralization on expected costs in a multi-location newsboy problem. Management Science, 25, 498-501. 
[17] Eynan, A. (1996). The impact of demands' correlation on the effectiveness of component commonality. International Journal of Production Research, 34, 1581-1602.

[18] Eynan, A. \& M. Rosenblatt (1996). Component commonality effects on inventory costs. IIE Transactions, 28, 93-104.

[19] Fisher, L. M., K. Ramdas \& K. T. Ulrich (1999). Component sharing in management of product variety: A study of automotive braking systems. Management Science, 45, 297-315.

[20] Gerchak, Y., M. J. Magazine \& A. B. Gamble (1988). Component commonality with service level requirements. Management Science, 34, 753-760.

[21] Gerchak, Y. \& M. Henig (1989) Component commonality in assemble-to-order systems: models and properties. Naval Research Logistics, 36, 61-68.

[22] Gupta, S. \& V. Krishnan (1999). Integrated component and supplier selection for a product family. Production and Operations Management. 8, 163-181.

[23] Hausman, W. H., H. L. Lee, \& A. X. Zhang (1998). Joint demand fulfillment probability in a multi-item inventory system with independent order-up-to policies. European Journal of Operational Research, 109, 646-659.

[24] Hillier, M. S. (1999). Component commonality in a multiple-period inventory model with service level constraints. International Journal of Production Research, 37, 2665-2683.

[25] Kapuscinski, R., R. Zhang, P. Carbonneau, R. Moore \& B. Reeves (2004). Inventory decisions in Dell's supply chain. Interfaces, 34, 191-205.

[26] Krishnan, V. \& S. Gupta (2001). Appropriateness and impact of platform based product development. Management Science, 47, 52-68.

[27] Krishnan, V. \& K. L. Ulrich (2001). Product development decisions: a review of the literature. Management Science, 47, 1-21.

[28] Lu, Y., Song, J. S. \& D. D. Yao (2003). Order fill rate, lead-time variability and advance demand information in an Assemble-to-order system. Operations Research, 51, 292-308.

[29] Lu, Y., Song, J. S. \& D. D. Yao (2005). Backorder minimization in multiproduct assemble-toorder systems. IIE Transactions, 37, 763-774. 
[30] Plambeck, E. and A. Ward (2003). A separation principle for a class of assemble-to-order systems with expediting. Operations Research (forthcoming).

[31] Plambeck, E. and A. Ward (2005). Optimal control of a high-volume assemble-to-order system. Working paper, Stanford University.

[32] Ramdas, K. (2003). Managing product variety: An integrated review and research directions. To appear in Production \& Operations Management.

[33] Rudi, N. (2000). Optimal inventory levels in systems with common components. Working Paper, The Simon School, University of Rochester. Rochester, NY.

[34] Simchi-Levi, D., P. Kaminsky \& E. Simchi-Levi (2003). Designing and Managing the Supply Chain: Concepts, Strategies, and Case Studies. McGraw-Hill/Irwin, NY.

[35] Song, J. S. (1998). On the order fill rate in a multi-item, base-stock inventory system. Operations Research, 46, 831-845.

[36] Song, J. S. (2000). A note on Assemble-to-Order systems with batch ordering. Management Sciences, 46, 739-743.

[37] Song, J. S. (2002). Order-Based backorders and their implications in multi-item inventory systems. Management Science, 48, 499-516.

[38] Song, J.-S., S. Xu and B. Liu (1999). Order-fulfillment performance measures in an assembleto-order system with stochastic leadtimes, Operations Research 47, 131-149.

[39] Song, J. S. \& D. D. Yao (2002). Performance analysis and optimization in Assemble-to-order systems with random lead-times. Operations Research, 50, 889-903.

[40] Song, J. S. \& P. Zipkin (2003). Supply chain operations: Assemble-to-order systems. Chapter 11 in Handbooks in Operations Research and Management Science, Vol. 11: Supply Chain Management.

[41] Swaminathan, J. M. \& S. Tayur (1998). Managing broader product lines through delayed differentiation using vanilla boxes. Management Science, 44, S161-S172.

[42] Thonemann, U. W. \& J. R. Bradley (2002). The effect of product variety on supply-chain performance. European Journal of Operational Research, 143, 548-569. 
[43] Thonemann, U. W. \& M. L. Brandeau (2000). Optimal commonality in component design. Operations Research, 48, 1-19.

[44] Ulrich, K. L. (1995). The role of product architecture in the manufacturing firm. Research Policy, 24, 419-440.

[45] Van Mieghem, J. A. (2004). Note-Commonality strategies: value drivers and equivalence with flexible capacity and inventory substitution. Management Science, 50, 419-424.

[46] Whyte, C. (2003). Motorola's battle with supply and demand chain complexity. iSource Business, April/May.

[47] Zhao, Y. \& D. Simchi-Levi (2006). Performance analysis and evaluation of Assemble-to-Order systems with stochastic sequential lead times. Operations Research, 54, 706-724.

[48] Zhang, A. X. (1997). Demand fulfillment rates in an assemble-to-order system with multiple products and dependent demands. Production and Operations Management, 6, 309-323. 